\title{
High triglyceride-glucose index is associated with adverse cardiovascular outcomes in patients with acute myocardial infarction
}

Yue Zhang

Capital Medical University

Xiaosong Ding

Capital Medical University

Bing Hua

Capital Medical University

Qingbo Liu

Capital Medical University

Hui Gao

Capital Medical University

Hui Chen

Capital Medical University

Xue-Qiao Zhao

University of Washington

Weiping Li

Capital Medical University

Hongwei Li ( $\nabla$ lhw19656@sina.com )

Capital Medical University

Original investigation

Keywords: triglyceride-glucose index (TyG index), acute myocardial infarction (AMI), major adverse cardiovascular events (MACEs)

Posted Date: April 27th, 2020

DOI: https://doi.org/10.21203/rs.3.rs-24155/v1

License: (1) (1) This work is licensed under a Creative Commons Attribution 4.0 International License.

Read Full License 
Version of Record: A version of this preprint was published at Nutrition, Metabolism and Cardiovascular Diseases on November 1st, 2020. See the published version at https://doi.org/10.1016/j.numecd.2020.07.041. 


\section{Abstract \\ Background}

Triglyceride glucose (TyG) index is considered a new marker for metabolic disorders. Although recent studies have found an association between TyG index level and vascular disease development, the prognostic value of TyG index in patients with acute myocardial infarction (AMI) remains unclear.

\section{Methods}

A total of 3181 patients with AMI, who underwent coronary angiography, were identified from the Cardiovascular Center of Beijing Friendship Hospital Database Bank and included in the analysis. Patients were stratified into 2 groups according to their baseline TyG index levels: the TyG index $<8.88$ group and the TyG index $\geq 8.88$ group. Clinical characteristics,biochemical parameters, and the incidence of major adverse cardiovascular events (MACEs) during a median of 33.3-month follow-up were recorded. The TyG index was calculated using the following formula: In [fasting triglycerides $(\mathrm{mg} / \mathrm{dL}) \times$ fasting plasma glucose $(\mathrm{mg} / \mathrm{dL}) / 2]$.

\section{Results}

Kaplan-Meier analysis revealed no significant difference in the incidence of all-cause death and cardiac death between the 2 groups. Compared with the TyG index $<8.88$ group, the TyG index $\geq 8.88$ group had significantly higher incidences of non-fatal $\mathrm{MI}$, revascularization, cardiac rehospitalization and composite MACEs. Multivariable Cox regression models revealed that the TyG index was positively associated with all-cause death [HR $(95 \% \mathrm{Cl}): 1.51(1.10,2.06), \mathrm{P}=0.010]$, cardiac death [HR $(95 \% \mathrm{Cl}): 1.68(1.19,2.38), \mathrm{P}=$ 0.004], revascularization [HR $(95 \% \mathrm{Cl}): 1.50(1.16,1.94), \mathrm{P}=0.002]$, cardiac rehospitalization [HR $(95 \% \mathrm{Cl})$ : 1.25 (1.05,1.49), $\mathrm{P}=0.012$ ], and composite MACEs [HR $(95 \% \mathrm{Cl}): 1.19(1.01,1.41), \mathrm{P}=0.046]$ in patients with AMI. The independent predictive effect of TyG index on all-cause death and cardiac death was mainly reflected in the subgroups of male gender, body mass index $\geq 25 \mathrm{~kg} / \mathrm{m}^{2}$, smoker, diabetes mellitus, estimated glomerular filtration rate (eGFR) $\geq 60 \mathrm{ml} / \mathrm{min} / 1.73 \mathrm{~m}^{2}$, high-density lipoprotein cholesterol $\geq 1.01 \mathrm{mmol} / \mathrm{L}$ and left ventricular ejection fraction (LVEF) $\geq 0.50$. The results also revealed that diabetes mellitus, previous AMI, eGFR, LVEF, and multi-vessel/left main coronary artery lesions were independent predictors of MACEs in patients with AMI (all $\mathrm{P}<0.05)$.

\section{Conclusions}

High TyG index levels appeared to be associated with an increased risk of MACEs in patients with AMI. The TyG index might be a valid predictor of cardiovascular outcomes of patients with AMI. 


\section{Trial registration:}

retrospectively registered

\section{Background}

Acute myocardial infarction (AMI) is the leading cause of morbidity and mortality of cardiovascular disease worldwide ${ }^{[1,2]}$. The World Bank estimated that the number of individuals with MI in China will increase to 23 million by $2030^{[3]}$. Moreover, a recent study has shown that AMI tends to occur in younger individuals ${ }^{[4]}$. This will undoubtedly put a huge strain on the health care system. Therefore, early risk stratification is critical to effectively prevent and manage AMI.

Insulin resistance (IR), an indicator of abnormal metabolism, not only contributes substantially to the pathogenesis of cardiovascular disease, but also correlates significantly with adverse cardiovascular outcomes ${ }^{[5-7]}$. The gold-standard test for IR evaluation is the hyperinsulinemic-euglycemic clamp ${ }^{[8]}$. Because the method is time-consuming and complex, the usage of the hyperinsulinemic-euglycemic clamp in large population studies and in the clinical setting remains to be difficult ${ }^{[9]}$.

The homeostasis model assessment of IR (HOMA-IR) has been proposed to predict IR and can be used in large-scale and epidemiological studies. However, because HOMA-IR depends on a plasma insulin assay, this method is expensive and is not available in most laboratories in underdeveloped areas. Clinicians need a simple and reliable index to quantitively evaluate IR. Kelley et al. have indicated that increased serum triglycerides (TGs) can impair muscle glucose metabolism and thus reduce insulin sensitivity [10]. Therefore, researchers began to study the triglyceride glucose (TyG) index, which combines TGs and fasting plasma glucose (FPG) levels, and found that the TyG index was a reproducible, reliable, and valid surrogate marker of IR ${ }^{[11-13]}$. In addition, the TyG index has been found to be well correlated with coronary artery calcification ${ }^{[14]}$, arterial stiffness ${ }^{[15]}$, carotid atherosclerosis ${ }^{[16]}$, coronary artery stenosis [17], and symptomatic coronary artery disease ${ }^{[18]}$. Su et al. indicated that TyG index appeared to be a stronger predictive factor than glycated hemoglobin (HbA1c) and TGs for cardiovascular events in patients with diabetes ${ }^{[19]}$. Luo et al. firstly reported an association between high TyG index levels and increased risk of major adverse cardiovascular and cerebrovascular events (MACCEs) of patients undergoing percutaneous coronary intervention (PCI) to treat ST-elevation myocardial infarction (STEMI) [20]. Mao et al. also found that in patients with non-ST-segment elevation acute coronary syndrome (NSTE-ACS), the patients with high TyG index showed significantly increased risk of major adverse cardiovascular events (MACEs) compared with the patients with low TyG index [21].

To the best of our knowledge, the relationship between TyG index and cardiovascular outcomes in patients with AMI, including both STEMI and Non-ST-segment elevation myocardial infarction (NSTEMI), is still unknown. Our study was to fill this knowledge gap. Here, we aimed to investigate the predictive value of TyG index on the cardiovascular outcomes of patients with AMI. In addition, to further delineate 
the risk stratification of AMI, we analyzed the relationship between the TyG index and all-cause death and cardiac death in different subgroups.

\section{Methods}

\section{Study population}

Study subjects were identified from the Cardiovascular Center of Beijing Friendship Hospital Database (CBD) Bank. The patient flowchart is presented in Figure 1. A total of 13,106 consecutive patients were diagnosed with ACS from January 2013 to July 2019. Of these 13106, 3307 were diagnosed with AMI and underwent coronary angiography. Of the 3307 patients, 126 were excluded according to the exclusion criteria, which were 1) with acute infectious disease, rheumatic disease, hematological disease, or neoplastic disease, 2) with severe valvulopathy or cardiomyopathy, 3) lacking clinical or follow-up data. Finally, 3181 patients were included in this analysis. According to the median value of TyG index level, 3181 patients were stratified into 2 groups (TyG index $<8.88$ group, $n=1601$ and TyG index $\geq 8.88$ group, $\mathrm{n}=1580$ ). All patients were followed up till October 31,2019 with a median follow up of 33.3 (IQR: 13.8, 49.8) months.

\section{Data collections and definitions}

The data collection protocol was approved by the Institutional Review Board of Beijing Friendship Hospital affiliated to Capital Medical University.

Patients' demographics, medical history, laboratory test results, echocardiographic, and angiographic evaluation results were collected and verified using an electronic medical recording system. The outcomes from MACEs were collected and recorded during clinical follow-up visits.

AMI, including STEMI and NSTEMI, was defined as chest pain with new ST-segment changes and elevation of myocardial necrosis markers to at least twice of the upper limit of the normal range. MACEs included all-cause death, non-fatal MI, revascularization, and cardiac rehospitalization (admission because of angina or heart failure). All-cause death was defined as the incidence of cardiac death or noncardiac death. Cardiac death was defined as fatal myocardial infarction, sudden death, and other cardiovascular death. Any coronary revascularization was defined as a revascularization of the target vessel or non-target vessels. Cardiac rehospitalization refers to rehospitalization for angina pectoris or heart failure. The TyG index was calculated as In [fasting TG $(\mathrm{mg} / \mathrm{dL}) \times$ fasting plasm glucose (FPG, $\mathrm{mg} / \mathrm{dL}$ )/2] ${ }^{[22]}$.

\section{Statistical analyses}

Continuous variables were presented as mean \pm standard deviation (SD) or median (IQR). Comparisons between the 2 groups were analyzed by Student's $t$-test or Mann-Whitney U-test. Categorical variables were expressed as number and percentage and compared using the Pearson chi-square test or Fisher's 
exact test. The cumulative incidence of MACEs was estimated by Kaplan-Meier survival curves, and the groups were compared using the log-rank test. A multivariable Cox regression analysis was performed in order to determine whether TyG index was an independent predictor for MACEs, and to identify other predictors of MACEs. Baseline variables that were significantly correlated with outcomes by univariate analysis and clinically relevant were entered into the multivariate model. Also, intercorrelations and colinearity among variables were taken into consideration in the multivariate analysis. All analyses were two-tailed and $P$ value $<0.05$ was considered statistically significant. Data were analyzed using IBM SPSS statistics 24 .

\section{Results}

\section{Patient characteristics}

As shown in Figure 1, of the 3181 eligible patients, 1601 patients with TyG index $<8.88$; 1580 patients with TyG index $\geq 8.88$. Comparing with the TyG index $<8.88$ group, patients in the TyG index $\geq 8.88$ group were significantly younger, showed lower percent of male, higher body mass index (BMI), lower percent of previous $\mathrm{AMI}$, and higher percent of diabetes mellitus and hypertension (all $p<0.05$ ). The TyG index $\geq 8.88$ group had significantly higher white cell count (WBC), hemoglobin, FPG, HbA1c, albumin, estimated glomerular filtration rate (eGFR), total cholesterol (TC), TGs and low-density lipoprotein cholesterol (LDL-C) than the TyG index $<8.88$ group (all $P<0.05$ ). However, the high-density lipoprotein cholesterol (HDL-C) level of TyG index $\geq 8.88$ group was significantly lower than that of TyG index $<8.88$ group $(p<0.001)$. Echo evaluation showed that the left ventricular ejection fraction (LVEF) were similar between the 2 groups. Angiographically, the TyG index $\geq 8.88$ group had significant higher percent of multi-vessel/left main (LM) coronary artery lesions than the TyG index $<8.88$ group $(p<0.001)$. Moreover, the proportion of patients receiving $\mathrm{PCl} /$ coronary artery bypass graft (CABG) during hospitalization was significantly higher in the TyG index $\geq 8.88$ group than in the TyG index $<8.88$ group $(p<0.05)$. Medication use during hospitalization were similar between the 2 groups except that significantly more patients treated with beta-blocker in the TyG index $\geq 8.88$ group than in the TyG index $<8.88$ group $(p<0.001$, Table 1).

\section{Risk factors for MACEs}

Univariate and multivariate analysis results and predictors for composite MACEs are presented in Table $\mathbf{2}$. Univariate analysis revealed that TyG index, age, male gender, systolic blood pressure (SBP), diabetes mellitus, hypertension, previous AMI, hemoglobin, FPG, HbA1c, albumin, creatinine, eGFR, TC, TGs, LDL-C, LVEF and multi-vessel/LM coronary artery lesions were risk factors for MACEs in patients with AMI (all $p$ $<0.05)$. The results of co-linearity analysis of MACEs predictors and TyG index are displayed in Table 3 . TC, LDL-C, and TyG index had high co-linearity. Therefore, TC and LDL-C were not included in the multivariate model. In addition, hypertension was significantly correlated with SBP $(r=0.303, p<0.001)$, diabetes mellitus was significantly correlated with FPG $(r=0.591, p<0.001)$ and $\mathrm{HbA1c}(\mathrm{r}=0.713, p<0.001)$, and eGFR was significantly correlated with creatinine $(r=-0.556, p<0.001)$. Therefore, SBP, FPG, HbA1c, 
and creatinine were also not included in the multivariate model. After adjusting for age and other potential confounding factors, multivariate analysis found that the TyG index, diabetes mellitus, previous AMI, eGFR, LVEF and multi-vessel/LM coronary artery lesions were independent predictors of MACEs in patients with AMI (all $p<0.05$, Table 2).

\section{Kaplan-Meier survival analysis for cardiovascular outcomes.}

During a median of 33.3-month (IQR 13.8, 49.8) follow-up, composite MACEs occurred in $407(25.4 \%)$ patients in TyG index $<8.88$ group and $449(28.4 \%)$ patients in TyG index $\geq 8.88$ group [unadjusted HR $(95 \% \mathrm{Cl}): 1.16(1.02,1.33), p=0.027]$. All-cause death occurred in $136(8.5 \%)$ patients in the low TyG index group and $113(7.2 \%)$ patients in the high TyG index group [unadjusted HR $(95 \% \mathrm{Cl}): 0.85(0.66,1.09), p=$ 0.205]. Cardiac death occurred in 100 (6.2\%) patients in the low TyG index group and 97 (6.1\%) patients in the high TyG index group [unadjusted HR $(95 \% \mathrm{Cl}): 0.99(0.75,1.31), p=0.948$ ]. Non-fatal MI occurred in $82(5.1 \%)$ patients in the low TyG index group and $113(7.2 \%)$ patients in the high TyG index group [unadjusted HR $(95 \% \mathrm{Cl}): 1.43(1.08,1.90), p=0.014$ ]. Revascularization occurred in $107(6.7 \%)$ patients in the low TyG index group and $182(11.5 \%)$ patients in the high TyG index group [unadjusted HR $(95 \% \mathrm{Cl})$ : $1.78(1.40,2.26), p<0.001]$. Cardiac rehospitalization occurred in $232(14.5 \%)$ patients in the low TyG index group and $283(17.9 \%)$ patients in the high TyG index group [unadjusted $\mathrm{HR}(95 \% \mathrm{Cl})$ :

1.27(1.07,1.51), $p=0.007$ ] (Figure 2).

\section{Independent association between TyG index and subsequent MACEs}

After adjusting for age, gender and other potential confounding factors, multivariate Cox regression analysis showed that the TyG index was independent predictors of all-cause death [HR $(95 \% \mathrm{Cl}): 1.51$ $(1.10,2.06), p=0.010]$, cardiac death [HR $(95 \% \mathrm{Cl}): 1.68(1.19,2.38), p=0.004]$, revascularization [HR (95\%Cl): $1.50(1.16,1.94), p=0.002]$, cardiac rehospitalization [HR (95\%Cl): $1.25(1.05,1.49), p=0.012$ ] and composite MACEs [HR (95\%Cl): $1.19(1.01,1.41), p=0.046$ ] in AMI patients (Table 4). Cumulative hazard curves adjusted for multiple variables for all-cause death and cardiac death of the 2 groups are presented in Figure 3.

\section{Independent association of TyG index with all-cause death and cardiac death in different subgroups}

The independent predictive effect of TyG index on all-cause death was mainly reflected in the subgroups of male gender, $\mathrm{BMI} \geq 25 \mathrm{~kg} / \mathrm{m}^{2}$, smoker, diabetes mellitus, eGFR $\geq 60 \mathrm{ml} / \mathrm{min} / 1.73 \mathrm{~m}^{2}, \mathrm{HDL}-\mathrm{C}$ $\geq 1.01 \mathrm{mmol} / \mathrm{L}$, and LVEF $\geq 0.50$ (Figure $4 a$ ). While the TyG index was significantly and independently associated with cardiac death in the subgroups of male gender, $\mathrm{BMI} \geq 25 \mathrm{~kg} / \mathrm{m}^{2}$, smoker, hypertension, diabetes mellitus, eGFR $\geq 60 \mathrm{ml} / \mathrm{min} / 1.73 \mathrm{~m}^{2}$, eGFR $<60 \mathrm{ml} / \mathrm{min} / 1.73 \mathrm{~m}^{2}, \mathrm{HDL}-\mathrm{C} \geq 1.01 \mathrm{mmol} / \mathrm{L}$, and LVEF $\geq 0.50$ (Figure 4b).

\section{Discussion}


To the best of our knowledge, this is the first study to explore the association between the TyG index and cardiovascular outcomes in patients with all types of AMI, especially in different subgroups. Our main findings include: (1) the TyG index was an independent predictor of all-cause death, cardiac death, revascularization, cardiac rehospitalization, and composite MACEs, and (2) the predictive effect of TyG index on all-cause death and cardiac death was mainly reflected in the subgroups of male gender, $\mathrm{BMI} \geq$ $25 \mathrm{~kg} / \mathrm{m}^{2}$, smoker, diabetes mellitus, eGFR $\geq 60 \mathrm{ml} / \mathrm{min} / 1.73 \mathrm{~m}^{2}, \mathrm{HDL}-\mathrm{C} \geq 1.01 \mathrm{mmol} / \mathrm{L}$, and LVEF $\geq$ 0.50 , and (3) Moreover, diabetes mellitus, previous AMI, eGFR, LVEF, and multi-vessel/LM coronary artery lesions were also independent predictors of MACEs in patients with AMI.

IR is defined as a decrease in the efficiency of insulin in promoting glucose uptake and utilization and is an indicator of abnormal metabolism. IR induces cardiovascular disease progression by several mechanisms [23]: (1) inducing glucose metabolism imbalance, which leads to chronic hyperglycemia and then in turn triggers oxidative stress and causes inflammatory responses; (2) altering systemic lipid metabolism, including increased TGs levels, decreased HDL-C levels, increased small dense low-density lipoproteins, and excessive postprandial lipemia; (3) causing endothelial dysfunction by decreasing nitric oxide production from endothelial cells and increasing procoagulant factor release.

Several previous studies found that IR was an important risk factor for cardiovascular disease and poor clinical outcomes ${ }^{[24-27]}$. Because the hyperinsulinemic-euglycemic clamp and the HOMA-IR were time consuming and costly, they cannot be used in clinic practice on a large scale. Thus, researchers began to study the TyG index, and found that it was a reproducible, reliable, and valid surrogate marker of IR $[12,13]$.

Recent studies have robustly proved that TyG index is closely related to an increased risk of diabetes and vascular disease. Zhang et al. showed that the cumulative risk of incident type 2 diabetes mellitus is increased as TyG index increases ${ }^{[28,29]}$. The TyG index level was positively correlated with the incidence of ischemic stroke ${ }^{[30,31]}$. In addition, previous studies have shown that a higher TyG index is significantly associated with a higher risk of coronary artery calcification ${ }^{[14]}$, arterial stiffness ${ }^{[15,32]}$, carotid atherosclerosis ${ }^{[16]}$, coronary artery stenosis ${ }^{[17]}$, and nephric microvascular damage ${ }^{[32]}$. Sánchez-línigo et al. suggested that the TyG index might be used to early identify the high-risk cardiovascular events in healthy individuals ${ }^{[33]}$. Da Silva et al. found that the TyG index was positively correlated with a higher prevalence of symptomatic coronary artery disease ${ }^{[18]}$, whereas Alizargar et al. believed that this result could be easily biased by diabetes and hyperlipidemia ${ }^{[34]}$. A recent study firstly confirmed that the TyG index was independently associated with SYNTAX score [OR (95\% Cl): $6.06(2.92,12.58), p<0$ 001] and MACEs [HR (95\% Cl): $1.79(1.05,3.07), p=0$ 034] in NSTE-ACS population ${ }^{[21]}$. Luo et al. found that in patients with STEMI undergoing $\mathrm{PCl}$, the incidence of composite MACCEs and all-cause death within 30 days, 6 months and 1 year were higher among those with highest level of TyG index (TyG index $\geq 9.608$ ), and that the TyG index $\geq 9.608$ was independently associated with an increased risk of MACCEs within 1 year [HR(95\% Cl): $1.53(1.01,2.06), p=0$ 003] ${ }^{[20]}$. However, the effects of the TyG index on cardiovascular outcomes in patients with all types of AMI, including STEMI and NSTEMI, are still unclear. 
In this study, we investigated the prognostic value of the TyG index in patients with all types of AMI for the first time. In addition, to better understand the predictive power of TyG index for different cardiovascular events, we analyzed the correlation between TyG index and each type of MACEs (including all-cause death, cardiac death, non-fatal MI, revascularization, and cardiac rehospitalization). In the current study, all-cause mortality and cardiac mortality in TyG index $<8.88$ group were $8.5 \%$ and $6.2 \%$, respectively, whereas those in TyG index $\geq 8.88$ group were $7.2 \%$ and $6.1 \%$, respectively. We attributed this "anomalous" result to the significant different baseline characteristics between the 2 groups. In this study, patients in the TyG index $\geq 8.88$ group were significantly younger, showed lower percent of male, lower percent of previous AMI, higher levels of hemoglobin, albumin and eGFR. In addition, the proportion of patients receiving $\mathrm{PCl} / \mathrm{CABG}$ and beta-blocker during hospitalization was significantly higher in the TyG index $\geq 8.88$ group than in the TyG index $<8.88$ group. Because of these protective factors, the all-cause mortality and cardiac mortality in the TyG index $\geq 8.88$ group was not significantly higher than those in the TyG index $<8.88$ group. Although there was no significant difference in the incidence of all-cause death and cardiac death between the 2 groups before adjusting for confounding factors, multivariate COX regression analysis found that the high TyG index was an independent predictor of all-cause death [adjusted HR (95\% Cl): $1.51(1.10,2.06), P=0.010$ ] and cardiac death [adjusted HR $(95 \% \mathrm{Cl}): 1.68$ $(1.19,2.38), P=0.004]$ after adjusting for age, gender and other confounding factors. Compared with the TyG index $<8.88$ group, the TyG index $\geq 8.88$ group had significantly higher incidences of non-fatal MI, revascularization, cardiac rehospitalization, and composite MACEs during the median of 33.3 months of follow-up. After adjusting for age, gender and other potential confounding factors, we also found that the high TyG index was an independent predictor of revascularization, cardiac rehospitalization, and composite MACEs.

The novelty of this study was the analysis of the predictive effect of TyG index on all-cause death and cardiac death in different subgroups for the first time. We found that the TyG index was significantly and independently associated with all-cause death and cardiac death in the subgroups of male gender, $\mathrm{BMI} \geq$ $25 \mathrm{~kg} / \mathrm{m}^{2}$, smoker, diabetes mellitus, eGFR $\geq 60 \mathrm{ml} / \mathrm{min} / 1.73 \mathrm{~m}^{2}, \mathrm{HDL}-\mathrm{C} \geq 1.01 \mathrm{mmol} / \mathrm{L}$, and LVEF $\geq$ 0.50. In addition, the independent predictive effect of TyG index on cardiac death was also reflected in the subgroups of hypertension and eGFR $<60 \mathrm{ml} / \mathrm{min} / 1.73 \mathrm{~m}^{2}$. This finding implied that using TyG index for early risk stratification in these subgroups may have important clinical significance.

Luo et al ${ }^{[20]}$ and Mao et al ${ }^{[21]}$ demonstrated that the TyG index was an independent predictor of cardiovascular events in STEMI and NSTE-ACS population with a cut-off value of the TyG $\geq 9.608$ for STEMI and TyG $\geq 8.805$ for NSTE-ACS. In this study, we proposed the cut-off point of TyG $\geq 8.88$. We found that TyG $\geq 8.88$ independently predicted the incidence of MACEs in patients with AMI.

Our study had several limitations. First, this was a single-center study although including a large sample size; thus, generalization of the findings should be cautious. Second, laboratory parameters were only measured once after hospital admission, which could cause potential bias due to measurement error. Third, this was a retrospective observational study. The information on the levels of TyG index during follow-up was limited. Prospective cohort studies are required to confirm our findings. 


\section{Conclusions}

In conclusion, the current study firstly demonstrated that high TyG index level was a strong independent predictor of an increased risk of MACEs in patients with AMI. In addition, the independent predictive effect of TyG index on all-cause death and cardiac death was mainly reflected in the subgroups of male gender, $\mathrm{BMI} \geq 25 \mathrm{~kg} / \mathrm{m}^{2}$, smoker, diabetes mellitus, eGFR $\geq 60 \mathrm{ml} / \mathrm{min} / 1.73 \mathrm{~m}^{2}, \mathrm{HDL}-\mathrm{C} \geq 1.01 \mathrm{mmol} / \mathrm{L}$, and LVEF $\geq 0.50$. Thus, the TyG index appears to be a reliable and valid predictor of clinical outcomes of patients with AMI, especially in subgroups of certain clinical characteristics.

\section{Abbreviations}

AMI: acute myocardial infarction; IR: insulin resistance; HOMA-IR: the homeostasis model assessment of insulin resistance; TGs: triglycerides; TyG: triglyceride-glucose index; FPG: fasting plasma glucose; HbA1c, glycated hemoglobin; MACCEs: major adverse cardiovascular and cerebrovascular events; PCl: percutaneous coronary intervention; STEMI: ST-elevation myocardial infarction; NSTE-ACS: Non-STsegment elevation acute coronary syndrome; MACEs: major adverse cardiovascular events; NSTEMI: NonST-segment elevation myocardial infarction; BMI: body mass index; WBC: white blood cell; eGFR: estimated glomerular filtration rate; TC: total cholesterol; LDL-C: low-density lipoprotein cholesterol; HDLC: high-density lipoprotein cholesterol; LVEF: left ventricular ejection fraction; LM: left main; CABG: coronary artery bypass graft; SBP: systolic blood pressure.

\section{Declarations}

\section{Ethics approval and consent to participate}

The study data collections were approved by the Institutional Review Board of Beijing Friendship Hospital affiliated to Capital Medical University, and written informed consent was obtained from all patients.

\section{Consent for publication}

Not applicable.

\section{Availability of data and materials}

The datasets used and/or analyzed during the current study are available from the corresponding author on reasonable request.

\section{Competing interests}

The authors declare that they have no competing interests.

\section{Funding}


This work was supported by the National Natural Science Foundation of China (Grant No. 81670315) and the Natural Science Foundation of Beijing (Grant No. 7172059).

\section{Authors' contributions}

YZ performed study, statistical analysis and wrote manuscript. XSD, BH, QBL and HG participated in study data collection. HC contributed discussion and edited manuscript. XQZ designed study and revised manuscript. WPL designed study, performed statistical analysis and edited manuscript. HWL provided funding support, designed study and reviewed manuscript. All authors read and approved the final manuscript.

\section{Acknowledgements}

We gratefully acknowledge the contributions of all staffs who work on the CBD Bank.

\section{References}

1. Benjamin EJ, Virani SS, Callaway CW, et al. Heart disease and stroke statistics-2018 update: a report from the American Heart Association. Circulation.2018; 137(12): e67-492.

2. Rodriguez F, Mahaffey KW. Management of patients with NSTE-ACS: a comparison of the recent AHA/ACC and ESC guidelines. Journal of the American College of Cardiology. 2016; 68(3): 313-21.

3. The World Bank. Toward a healthy and harmonious life in China: stemming the rising tide of noncommunicable diseases. http://www.worldbank. org/content/ dam/Worldbank/document/NCD_report_en.pdf

4. Mercado-Lubo R, Yarzebski J, Lessard D, et al. Changing Trends in the Landscape of Patients Hospitalized With Acute Myocardial Infarction (2001 to 2011) (from the Worcester Heart Attack Study). Am J Cardiol.2020;125(5):673-7.

5. Laakso M, Kuusisto J. Insulin resistance and hyperglycaemia in cardiovascular disease development. Nature Reviews Endocrinologyvol.2014;10(5):293-302.

6. Eddy D, Schlessinger L, Kahn R, et al. Relationship of insulin resistance and related metabolic variables to coronary artery disease: a mathematical analysis. Diabetes Care.2009;32(2):361-6.

7. Caccamo G, Bonura F, Bonura F, et al.Insulin resistance and acute coronary syndrome.Atherosclerosis.2010; 211(2): 672-5.

8. DeFronzo RA, Tobin JD, Andres R. Glucose clamp technique: a method for quantifying insulin secretion and resistance. Am J Physiol.1979;237:e214-23.

9. Muniyappa R, Lee S, Chen H, Quon MJ. Current approaches for assessing insulin sensitivity and resistance in vivo: advantages, limitations, and appropriate usage. American Journal of PhysiologyEndocrinology and Metabolism.2008; 294(1) e15-26.

10. Kelley DE, Goodpaster BH. Skeletal muscle triglyceride. An aspect of regional adiposity and insulin resistance. Diabetes Care. 2001;24(5):933-41. 
11. Du TT, Yuan G, Zhang MX, et al. Clinical usefulness of lipid ratios, visceral adiposity indicators, and the triglycerides and glucose index as risk markers of insulin resistance. Cardiovasc Diabetol. 2014;13:146.

12. Guerrero-Romero F, Simental-Mendia LE, Gonzalez-Ortiz M, et al. The product of triglycerides and glucose, a simple measure of insulin sensitivity. Comparison with the euglycemichyperinsulinemic clamp. J Clin Endocrinol Metab. 2010;95:3347-51.

13. Bastard JP, Lavoie ME, Messier V, et al. Evaluation of two new surrogate indices including parameters not using insulin to assess insulin sensitivity/resistance in non-diabetic postmenopausal women: a MONET group study. Diabetes Metab. 2012;38:258-63.

14. Park K, Ahn CW, Lee SB, et al. Elevated TyG Index Predicts Progression of Coronary Artery Calcification. Diabetes Care.2019;42:1569-73.

15. Lee SB, Ahn CW, Lee BK, et al. Association between triglyceride glucose index and arterial stiffness in Korean adults. Cardiovasc Diabetol. 2018;17:41.

16. Irace C, Carallo C, Scavelli FB, et al. Markers of insulin resistance and carotid atherosclerosis. A comparison of the homeostasis model assessment and triglyceride glucose index. Int $\mathrm{J}$ Clin Pract. 2013;67(7):665-72.

17. Lee EY, Yang HK, Lee J, et al. Triglyceride glucose index, a marker of insulin resistance, is associated with coronary artery stenosis in asymptomatic subjects with type 2 diabetes. Lipids in Health and Disease.2016;15:155.

18. da Silva A, Caldas APS, Hermsdorff HHM, et al. Triglyceride-glucose index is associated with symptomatic coronary artery disease in patients in secondary care. Cardiovasc Diabetol.2019;18:89.

19. Su WF, Chen SC, Huang YT, et al. Comparison of the Effects of Fasting Glucose, Hemoglobin A1C, and Triglyceride-Glucose Index on Cardiovascular Events in Type 2 Diabetes Mellitus.2019; doi:10.3390/nu11112838.

20. Luo E, Wang D, Yan GL, et al. High triglyceride-glucose index is associated with poor prognosis in patients with acute ST-elevation myocardial infarction after percutaneous coronary intervention. Cardiovasc Diabetol. 2019;18:150.

21. Mao Q, Zhou DL, Li YM, et al.The Triglyceride-Glucose Index Predicts Coronary Artery Disease Severity and Cardiovascular Outcomes in Patients with Non-ST Segment Elevation Acute Coronary Syndrome. Disease Markers.2019; doi: 10.1155/2019/6891537.

22. Guerrero-Romero F, Simental-Mendía LE, González-Ortiz M, et al. The product of triglycerides and glucose, a simple measure of insulin sensitivity. Comparison with the euglycemic-hyperinsulinemic clamp.The Journal of Clinical Endocrinology \& Metabolism.2010; 95(7): 3347-51.

23. Ormazabal V, Nair S, Elfeky O, et al. Association between insulin resistance and the development of cardiovascular disease. Cardiovasc Diabetol.2018;17:122.

24. Reaven G. Insulin resistance and coronary heart disease in nondiabetic individuals. Arterioscler Thromb Vasc Biol. 2012;32(8):1754-9. 
25. Laakso M, Kuusisto J. Insulin resistance and hyperglycaemia in cardiovascular disease development. Nat Rev Endocrinol. 2014;10(5):293-302.

26. Bloomgarden ZT. Insulin resistance, dyslipidemia, and cardiovascular disease. Diabetes Care. 2007;30(8):2164-70.

27. Rutter MK, Meigs JB, Sullivan LM, et al. Insulin resistance, the metabolic syndrome, and incident cardiovascular events in the Framingham Offspring Study. Diabetes.2005; 54(11):3252-7.

28. Zhang $M$, Wang $B$, Liu $Y$, et al. Cumulative increased risk of incident type 2 diabetes mellitus with increasing triglyceride glucose index in normal-weight people: The Rural Chinese Cohort Study. Cardiovasc Diabetol. 2017; 16(1):30.

29. Navarro-González D, Sánchez-Î́igo L, Pastrana-Delgado J, et al. Triglyceride-glucose index (TyG index) in comparison with fasting plasma glucose improved diabetes prediction in patients with normal fasting glucose: The Vascular-Metabolic CUN cohort. Preventive Medicine.2016;86:99-105.

30. Sánchez-Íñigo L, Navarro- González D, Fernández-Montero A, et al. Risk of incident ischemic stroke according to the metabolic health and obesity states in the Vascular-Metabolic CUN cohort. International Journal of Stroke. 2017; 12(2): 187-91.

31. Shi W, Xing L, Jing L, et al. Value of triglyceride-glucose index for the estimation of ischemic stroke risk: Insights from a general population. Nutr Metab Cardiovasc Dis.2020;30(2):245-53.

32. Zhao S, Yu S, Chi C, et al. Association between macro-and microvascular damage and the triglyceride glucose index in community-dwelling elderly individuals: the Northern Shanghai Study. Cardiovasc Diabetol.2019;18(1):95.

33. Sánchez-Î́igo S, Navarro-González D, Fernández-Montero A, et al. The TyG index may predict the development of cardiovascular events. Eur J Clin Invest. 2016; 46 (2): 189-97.

34. Alizargar J, Bai CH, Hsieh NC, Wu SV. Use of the triglyceride-glucose index (TyG) in cardiovascular disease patients. Cardiovasc Diabetol.2020;19(1):8.

\section{Tables}


Table 1 Clinical characteristics of the 2 groups

\begin{tabular}{|c|c|c|c|}
\hline & TyG $<8.88(n=1601)$ & TyG $\geq 8.88(n=1580)$ & $\mathrm{P}$ value \\
\hline TyG index & $8.4 \pm 0.3$ & $9.5 \pm 0.5$ & $<0.001$ \\
\hline Age, years & $65.5 \pm 11.6$ & $61.1 \pm 11.9$ & $<0.001$ \\
\hline Male gender & $1253(78.3)$ & $1156(73.2)$ & 0.001 \\
\hline BMI, $\mathrm{kg} / \mathrm{m}^{2}$ & $24.9 \pm 3.4$ & $26.4 \pm 3.4$ & $<0.001$ \\
\hline $\mathrm{SBP}, \mathrm{mmHg}$ & $127.9 \pm 21.0$ & $129.7 \pm 22.8$ & 0.051 \\
\hline $\mathrm{DBP}, \mathrm{mmHg}$ & $74.4 \pm 12.8$ & $74.6 \pm 13.3$ & 0.093 \\
\hline \multicolumn{4}{|l|}{ Medical history } \\
\hline Current/ex-Smoker & $1007(62.9)$ & $1019(64.5)$ & 0.349 \\
\hline Diabetes mellitus & $375(23.4)$ & $856(54.2)$ & $<0.001$ \\
\hline Hypertension & $1069(66.8)$ & $1113(70.4)$ & 0.026 \\
\hline Previous AMI & $246(15.4)$ & 182(11.5) & 0.001 \\
\hline \multicolumn{4}{|l|}{ Laboratory values } \\
\hline $\mathrm{WBC}, 10^{9} / \mathrm{L}$ & $8.1 \pm 2.7$ & $9.1 \pm 3.1$ & $<0.001$ \\
\hline Neutrophil ratio, \% & $70.1 \pm 13.0$ & $70.0 \pm 13.3$ & 0.956 \\
\hline Hemoglobin, g/L & $135.7 \pm 17.6$ & $140.9 \pm 18.4$ & $<0.001$ \\
\hline $\mathrm{HsCRP}, \mathrm{mg} / \mathrm{L}$ & $6.4(1.9,16.6)$ & $7.5(2.6,14.7)$ & 0.121 \\
\hline $\mathrm{FPG}, \mathrm{mmol} / \mathrm{L}$ & $5.5 \pm 1.3$ & $8.1 \pm 3.2$ & $<0.001$ \\
\hline HbA1c, \% & $6.0 \pm 1.0$ & $7.1 \pm 1.8$ & $<0.001$ \\
\hline Albumin, g/L & $36.8 \pm 3.8$ & $38.3 \pm 4.0$ & $<0.001$ \\
\hline Creatinine, umol/L & $81.3(71.4,93.5)$ & $80.9(70.1,93.3)$ & 0.225 \\
\hline $\mathrm{eGFR}, \mathrm{ml} / \mathrm{min} / 1.73 \mathrm{~m}^{2}$ & $83.2(68.2,97.3)$ & $85.9(69.6,100.5)$ & 0.001 \\
\hline $\mathrm{TC}, \mathrm{mmol} / \mathrm{L}$ & $4.1 \pm 0.9$ & $4.8 \pm 1.1$ & $<0.001$ \\
\hline TGs, mmol/L & $1.1 \pm 0.3$ & $2.5 \pm 1.5$ & $<0.001$ \\
\hline LDL-C, mmol/L & $2.4 \pm 0.7$ & $2.8 \pm 0.8$ & $<0.001$ \\
\hline $\mathrm{HDL}-\mathrm{C}, \mathrm{mmol} / \mathrm{L}$ & $1.1 \pm 0.3$ & $1.0 \pm 0.2$ & $<0.001$ \\
\hline \multicolumn{4}{|l|}{ Echocardiography } \\
\hline LVEF & $0.59 \pm 0.10$ & $0.59 \pm 0.09$ & 0.553 \\
\hline \multicolumn{4}{|l|}{ Angiography findings } \\
\hline Multi-vessel/LM & $1199(74.9)$ & $1265(80.1)$ & $<0.001$ \\
\hline Proximal LAD & $621(38.8)$ & $620(39.2)$ & 0.794 \\
\hline \multicolumn{4}{|l|}{ In-hospital treatment } \\
\hline PCI/CABG & $1420(88.7)$ & $1444(91.4)$ & 0.011 \\
\hline Antiplatelet agent & 1572(98.2) & 1558(98.6) & 0.347 \\
\hline $\mathrm{ACEI} / \mathrm{ARB}$ & $1150(71.8)$ & $1177(74.5)$ & 0.090 \\
\hline Beta-blocker & 1291(80.6) & $1360(86.1)$ & $<0.001$ \\
\hline Statin & 1462(91.3) & $1422(90.0)$ & 0.201 \\
\hline
\end{tabular}

Values are presented as mean $\pm \mathrm{SD}$, median (IQR) or number (\%).

TyG, triglyceride-glucose index; BMI, body mass index; SBP, systolic blood pressure; DBP, diastolic blood pressure; AMI, acute myocardial infarction; WBC, white blood cell; HsCRP: high sensitivity C reactive protein; FPG, fasting plasma glucose; HbA1c, glycated hemoglobin; eGFR, 
estimated glomerular filtration rate; TC, total cholesterol; TGs, triglycerides; LDL-C, low-density lipoprotein cholesterol; HDL-C, high-density lipoprotein cholesterol; LVEF, left ventricular ejection fraction; LM, left main coronary artery; LAD, left anterior descending; PCI, percutaneous coronary intervention; CABG, coronary artery bypass graft; ACEI/ARB, angiotensin-converting enzyme inhibitor/angiotensin receptor blocker. 
Table 2 Results of univariate and multivariate analysis and predictors of composite MACEs

\begin{tabular}{|c|c|c|c|c|}
\hline Variable & $\begin{array}{r}\text { Univariate analysis } \\
\text { HR }(95 \% \mathrm{CI})\end{array}$ & $\mathrm{P}$ value & $\begin{array}{c}\text { Multivariate analysis } \\
\text { HR(95\% CI) }\end{array}$ & $\mathrm{P}$ value \\
\hline TyG index & $1.16(1.02,1.33)$ & 0.027 & $1.19(1.01,1.41)$ & 0.046 \\
\hline Age, years & $1.02(1.01,1.03)$ & $<0.001$ & $1.01(0.99,1.02)$ & 0.219 \\
\hline Male gender & $0.85(0.73,0.99)$ & 0.038 & $0.99(0.83,1.18)$ & 0.888 \\
\hline BMI, $\mathrm{kg} / \mathrm{m}^{2}$ & $1.02(0.89,1.17)$ & 0.740 & & \\
\hline $\mathrm{SBP}, \mathrm{mmHg}$ & $1.02(1.01,1.03)$ & 0.006 & & \\
\hline $\mathrm{DBP}, \mathrm{mmHg}$ & $1.01(0.99,1.02)$ & 0.227 & & \\
\hline \multicolumn{5}{|l|}{ Medical history } \\
\hline Current/ex-Smoker & $0.96(0.84,1.10)$ & 0.576 & & \\
\hline Diabetes mellitus & $1.36(1.19,1.56)$ & $<0.001$ & $1.16(1.01,1.34)$ & 0.047 \\
\hline Hypertension & $1.43(1.23,1.67)$ & $<0.001$ & $1.01(1.00,1.02)$ & 0.091 \\
\hline Previous AMI & $1.56(1.31,1.85)$ & $<0.001$ & $1.27(1.06,1.52)$ & 0.009 \\
\hline \multicolumn{5}{|l|}{ Laboratory values } \\
\hline WBC, $10^{9} / \mathrm{L}$ & $0.98(0.96,1.01)$ & 0.174 & & \\
\hline Neutrophil ratio, \% & $0.99(0.98,1.01)$ & 0.688 & & \\
\hline Hemoglobin, $\mathrm{g} / \mathrm{L}$ & $0.98(0.97,0.99)$ & 0.001 & $1.00(0.99,1.01)$ & 0.581 \\
\hline HsCRP, mg/L & $1.01(0.99,1.02)$ & 0.313 & & \\
\hline FPG, $\mathrm{mmol} / \mathrm{L}$ & $1.06(1.04,1.08)$ & $<0.001$ & & \\
\hline HbA1c, \% & $1.11(1.06,1.15)$ & $<0.001$ & & \\
\hline Albumin, $g / L$ & $0.97(0.95,0.98)$ & $<0.001$ & $0.99(0.97,1.01)$ & 0.252 \\
\hline Creatinine, umol/L & $1.02(1.01,1.03)$ & $<0.001$ & & \\
\hline $\mathrm{eGFR}, \mathrm{ml} / \mathrm{min} / 1.73 \mathrm{~m}^{2}$ & $0.98(0.97,0.99)$ & $<0.001$ & $0.98(0.97,0.99)$ & $<0.001$ \\
\hline $\mathrm{TC}, \mathrm{mmol} / \mathrm{L}$ & $0.80(0.71,0.91)$ & 0.001 & & \\
\hline TGs, mmol/L & $0.68(0.58,0.81)$ & $<0.001$ & $0.98(0.92,1.05)$ & 0.644 \\
\hline $\mathrm{LDL}-\mathrm{C}, \mathrm{mmol} / \mathrm{L}$ & $0.80(0.68,0.96)$ & 0.013 & & \\
\hline HDL-C, mmol/L & $1.10(0.83,1.44)$ & 0.512 & & \\
\hline \multicolumn{5}{|l|}{ Echocardiography } \\
\hline LVEF & $0.98(0.97,0.99)$ & $<0.001$ & $0.98(0.97,0.99)$ & $<0.001$ \\
\hline \multicolumn{5}{|l|}{ Angiography findings } \\
\hline Multi-vessel/LM & $1.99(1.64,2.42)$ & $<0.001$ & $1.63(1.33,2.00)$ & $<0.001$ \\
\hline Proximal LAD & $1.11(0.97,1.28)$ & 0.124 & & \\
\hline \multicolumn{5}{|l|}{ In-hospital treatment } \\
\hline PCI/CABG & $0.89(0.71,1.10)$ & 0.276 & & \\
\hline Antiplatelet agent & $0.73(0.39,1.36)$ & 0.317 & & \\
\hline ACEI/ARB & $0.93(0.73,1.18)$ & 0.539 & & \\
\hline
\end{tabular}

Page 16/22 
Adjusted factors included age, gender, diabetes mellitus, hypertension, previous AMI, hemoglobin, albumin, eGFR, TGs, LVEF and multi-vessel/LM. TyG, triglyceride-glucose index; BMI, body mass index; SBP, systolic blood pressure; DBP, diastolic blood pressure; AMI, acute myocardial infarction; WBC, white blood cell; HsCRP: high sensitivity C reactive protein; FPG, fasting plasma glucose; HbA1c, glycated hemoglobin; eGFR, estimated glomerular filtration rate; TC, total cholesterol; TGs, triglycerides; LDL-C, low-density lipoprotein cholesterol; HDL-C, high-density lipoprotein cholesterol; LVEF, left ventricular ejection fraction; LM, left main coronary artery; LAD, left anterior descending; PCI, percutaneous coronary intervention; CABG, coronary artery bypass graft; ACEI/ARB, angiotensin-converting enzyme inhibitor/angiotensin receptor blocker; HR, hazard ratio; CI, confidence interval.

Table 3 Co-linearity analysis of MACEs predictors and TyG index.

\begin{tabular}{|c|c|c|c|c|c|c|c|}
\hline & \multirow{2}{*}{\multicolumn{2}{|c|}{$\begin{array}{l}\text { Unstandardized } \\
\text { coefficients }\end{array}$}} & \multirow{2}{*}{\multicolumn{2}{|c|}{$\begin{array}{l}\text { Standardized } \\
\text { coefficients }\end{array}$}} & \multirow[t]{3}{*}{ Sig. } & \multirow{2}{*}{\multicolumn{2}{|c|}{$\begin{array}{l}\text { Collinearity } \\
\text { statistics }\end{array}$}} \\
\hline & & & & & & & \\
\hline & $\mathrm{B}$ & Std. error & Beta & & & Tolerance & VIF \\
\hline (Constant) & 2.644 & 0.152 & & 17.384 & $<0.001$ & & \\
\hline Age,years & 0.002 & 0.001 & 0.050 & 2.748 & 0.006 & 0.529 & 1.889 \\
\hline Gender & -0.084 & 0.019 & -0.072 & -4.446 & $<0.001$ & 0.668 & 1.496 \\
\hline $\mathrm{SBP}, \mathrm{mmHg}$ & $<0.001$ & $<0.001$ & 0.018 & 1.317 & 0.188 & 0.883 & 1.132 \\
\hline Diabetes mellitus & 0.089 & 0.019 & 0.087 & 4.725 & $<0.001$ & 0.517 & 1.936 \\
\hline Hypertension & 0.033 & 0.015 & 0.031 & 2.151 & 0.032 & 0.858 & 1.165 \\
\hline Previous AMI & -0.034 & 0.020 & -0.023 & -1.687 & 0.092 & 0.916 & 1.092 \\
\hline Hemoglobin, g/L & -0.001 & $<0.001$ & -0.051 & -3.015 & 0.003 & 0.603 & 1.659 \\
\hline FPG, mmol/L & -0.058 & 0.004 & -0.321 & -16.230 & $<0.001$ & 0.444 & 2.252 \\
\hline HbA1c, \% & -0.002 & 0.007 & -0.007 & -0.320 & 0.749 & 0.367 & 2.723 \\
\hline Albumin, $\mathrm{g} / \mathrm{L}$ & -0.011 & 0.002 & -0.091 & -5.965 & $<0.001$ & 0.745 & 1.342 \\
\hline Creatinine, umol/L & $<0.001$ & $<0.001$ & 0.021 & 1.192 & 0.233 & 0.573 & 1.746 \\
\hline $\mathrm{eGFR}, \mathrm{ml} / \mathrm{min} / 1.73 \mathrm{~m}^{2}$ & 0.001 & $<0.001$ & 0.052 & 2.467 & 0.014 & 0.391 & 2.561 \\
\hline $\mathrm{TC}, \mathrm{mmol} / \mathrm{L}$ & 0.087 & 0.022 & 0.184 & 3.986 & $<0.001$ & 0.082 & 12.248 \\
\hline TGs, mmol/L & -0.155 & 0.006 & -0.403 & -25.014 & $<0.001$ & 0.669 & 1.496 \\
\hline LDL-C, mmol/L & -0.186 & 0.028 & -0.289 & -6.549 & $<0.001$ & 0.089 & 11.210 \\
\hline LVEF & -0.001 & 0.001 & -0.029 & -2.039 & 0.042 & 0.886 & 1.128 \\
\hline Multi-vessel/LM & 0.032 & 0.017 & 0.027 & 1.938 & 0.053 & 0.899 & 1.112 \\
\hline
\end{tabular}

Dependent variable: TyG index

Co-linearity analysis showed that TC, LDL-C and TyG index had high co-linearity.

TyG, triglyceride-glucose index; SBP, systolic blood pressure; AMI, acute myocardial infarction; FPG, fasting plasma glucose; HbA1c, glycated hemoglobin; eGFR, estimated glomerular filtration rate; TC, total cholesterol; TGs, triglycerides; LDL-C, low-density lipoprotein cholesterol; LVEF, left ventricular ejection fraction; LM, left main coronary artery. 
Table 4 Multivariable Cox regression analysis of MACEs

\begin{tabular}{|c|c|c|c|c|c|c|}
\hline \multicolumn{7}{|c|}{$\mathrm{HR}(95 \% \mathrm{CI})$} \\
\hline & No adjustment & p value & Model $1^{\mathrm{a}}$ & $\mathrm{p}$ value & Model $2^{\mathrm{b}}$ & $\mathrm{p}$ value \\
\hline \multicolumn{7}{|c|}{ All cause death } \\
\hline TyG $<8.88$ & 1.0 & $-/-$ & 1.0 & $-1-$ & 1.0 & $-1-$ \\
\hline TyG $\geq 8.88$ & $0.85(0.66,1.09)$ & 0.205 & $1.14(0.88,1.47)$ & 0.320 & $1.51(1.10,2.06)$ & 0.010 \\
\hline \multicolumn{7}{|c|}{ Cardiac death } \\
\hline TyG $<8.88$ & 1.0 & $-1-$ & 1.0 & $-1-$ & 1.0 & $-1-$ \\
\hline TyG $\geq 8.88$ & $0.99(0.75,1.31)$ & 0.948 & $1.32(0.99,1.75)$ & 0.056 & $1.68(1.19,2.38)$ & 0.004 \\
\hline \multicolumn{7}{|c|}{ Non-fatal MI } \\
\hline TyG $<8.88$ & 1.0 & $-1-$ & 1.0 & $-1-$ & 1.0 & $-1-$ \\
\hline TyG $\geq 8.88$ & $1.43(1.08,1.90)$ & 0.014 & $1.58(1.19,2.11)$ & 0.002 & $1.29(0.95,1.75)$ & 0.100 \\
\hline \multicolumn{7}{|c|}{ Revascularization } \\
\hline TyG $<8.88$ & 1.0 & $-1-$ & 1.0 & $-1-$ & 1.0 & $-1-$ \\
\hline TyG $\geq 8.88$ & $1.78(1.40,2.26)$ & $<0.001$ & $1.67(1.23,2.03)$ & 0.001 & $1.50(1.16,1.94)$ & 0.002 \\
\hline \multicolumn{7}{|c|}{ Cardiac rehospitalization } \\
\hline TyG $<8.88$ & 1.0 & $-1-$ & 1.0 & $-1-$ & 1.0 & $-1-$ \\
\hline TyG $\geq 8.88$ & $1.27(1.07,1.51)$ & 0.007 & $1.26(1.06,1.50)$ & 0.009 & $1.25(1.05,1.49)$ & 0.012 \\
\hline \multicolumn{7}{|c|}{ Composite MACEs } \\
\hline TyG $<8.88$ & 1.0 & $-1-$ & 1.0 & $-1-$ & 1.0 & $-1-$ \\
\hline TyG $\geq 8.88$ & $1.16(1.02,1.33)$ & 0.027 & $1.26(1.10,1.44)$ & 0.001 & $1.19(1.01,1.41)$ & 0.046 \\
\hline
\end{tabular}

a Adjusted for age and gender.

b Adjusted for all covariates(age, gender, diabetes mellitus, hypertension, previous AMI, hemoglobin, albumin, eGFR, TGs, LVEF and multivessel/LM)

MACEs, major adverse cardiac events; TyG, triglyceride-glucose index; MI, myocardial infarction; AMI: acute myocardial infarction; eGFR, estimated glomerular filtration rate; TGs, triglycerides; LVEF: left ventricular ejection fraction; LM, left main coronary artery; HR, hazard ratio; CI, confidence interval.

\section{Figures}




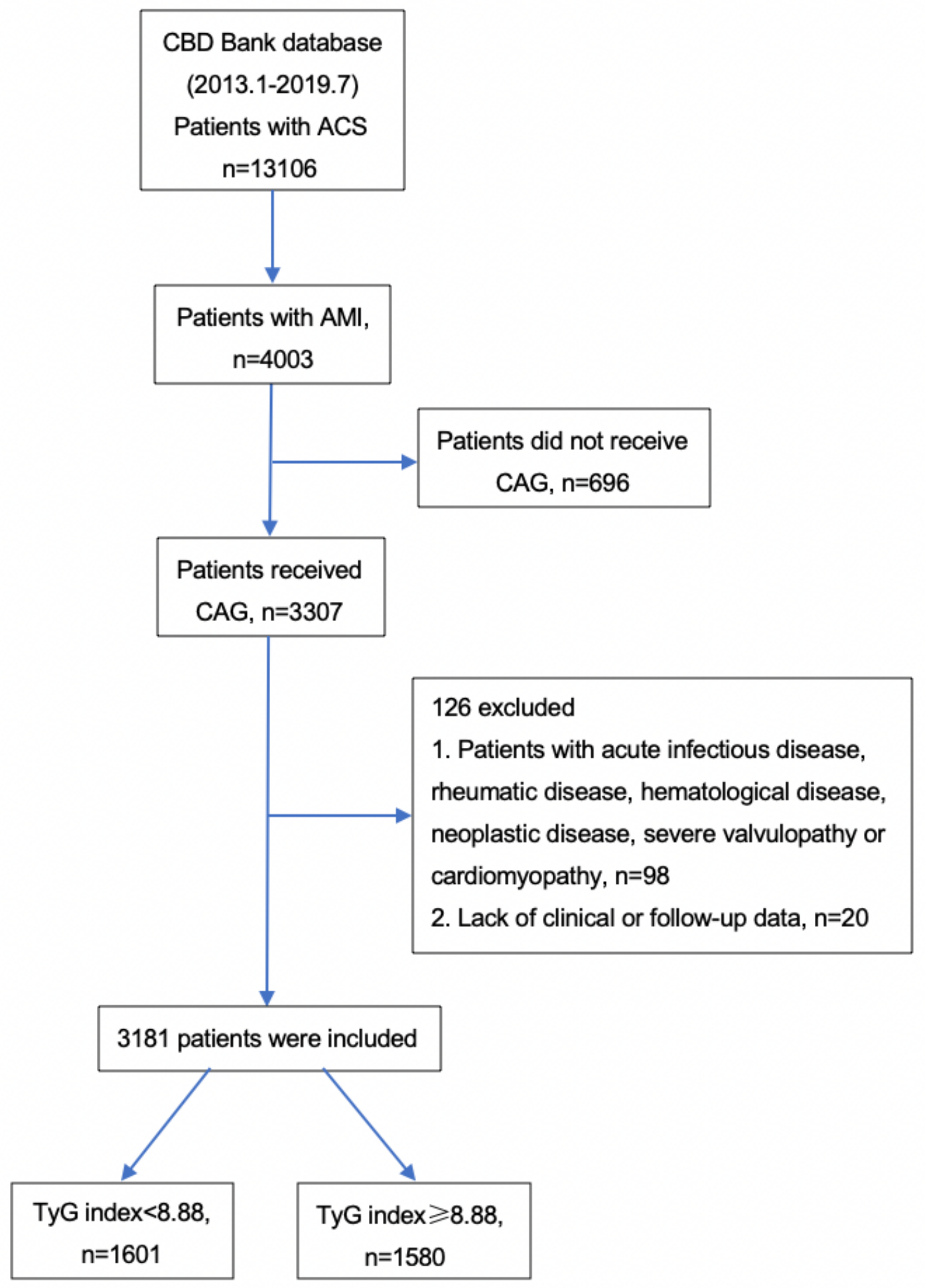

\section{Figure 1}

The flow chart of study subject enrollment. CBD, Cardiovascular Center of Beijing Friendship Hospital Database; ACS, acute coronary syndrome; AMI, acute myocardial infarction; CAG, coronary angiography; TyG, triglyceride-glucose index. 

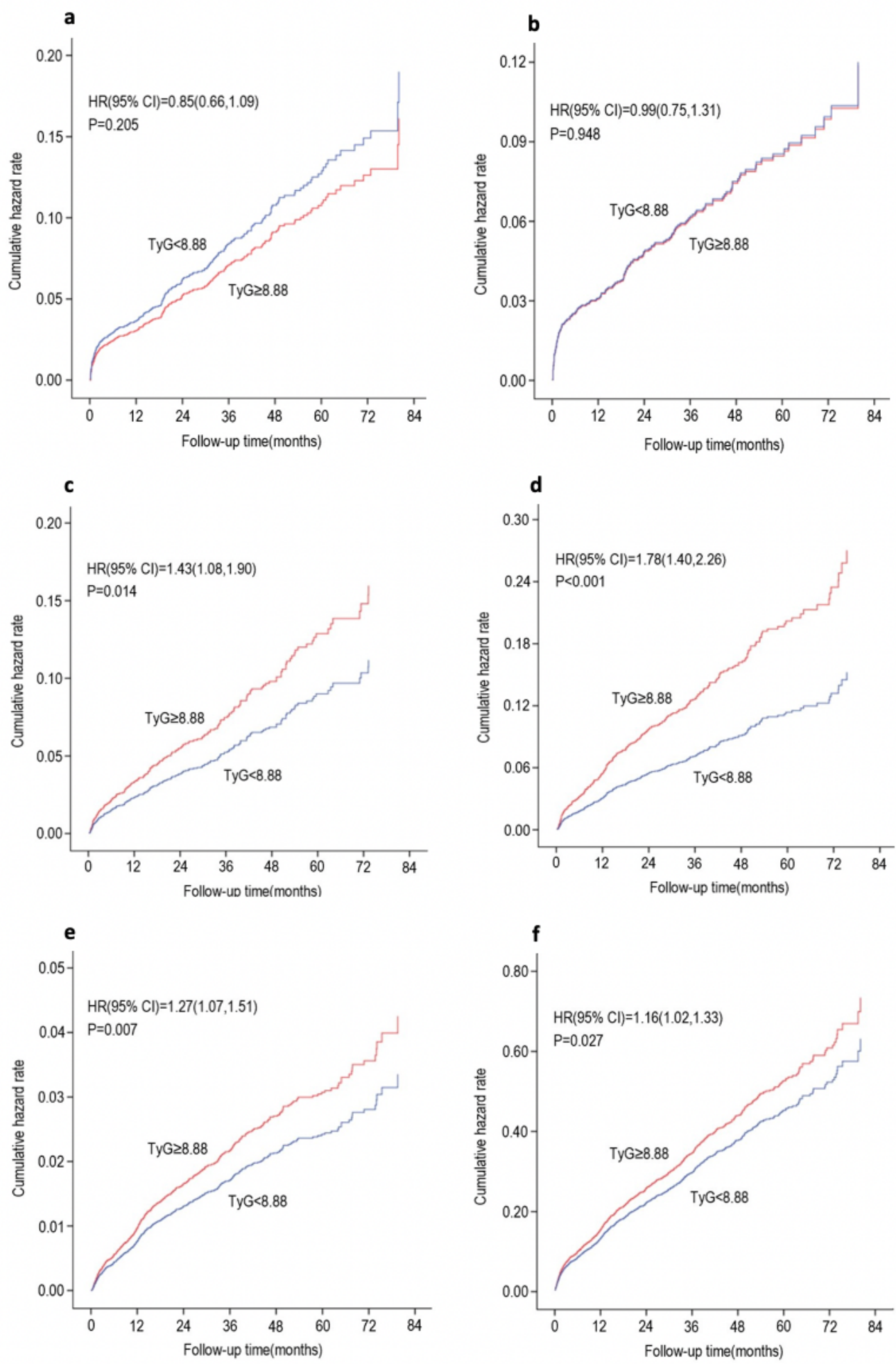

\section{Figure 2}

Kaplan-Meier curves for all-cause death(a), cardiac death(b), non-fatal MI(c), revascularization(d), cardiac rehospitalization(e) and composite MACEs(f) of the TyG<8.88 group(blue line) versus the TyG $\geq 8.88$ group(red line). TyG, triglyceride-glucose index; MI, myocardial infarction; MACEs, major adverse cardiac events; $\mathrm{HR}$, hazard ratio; $\mathrm{Cl}$, confidence interval. 

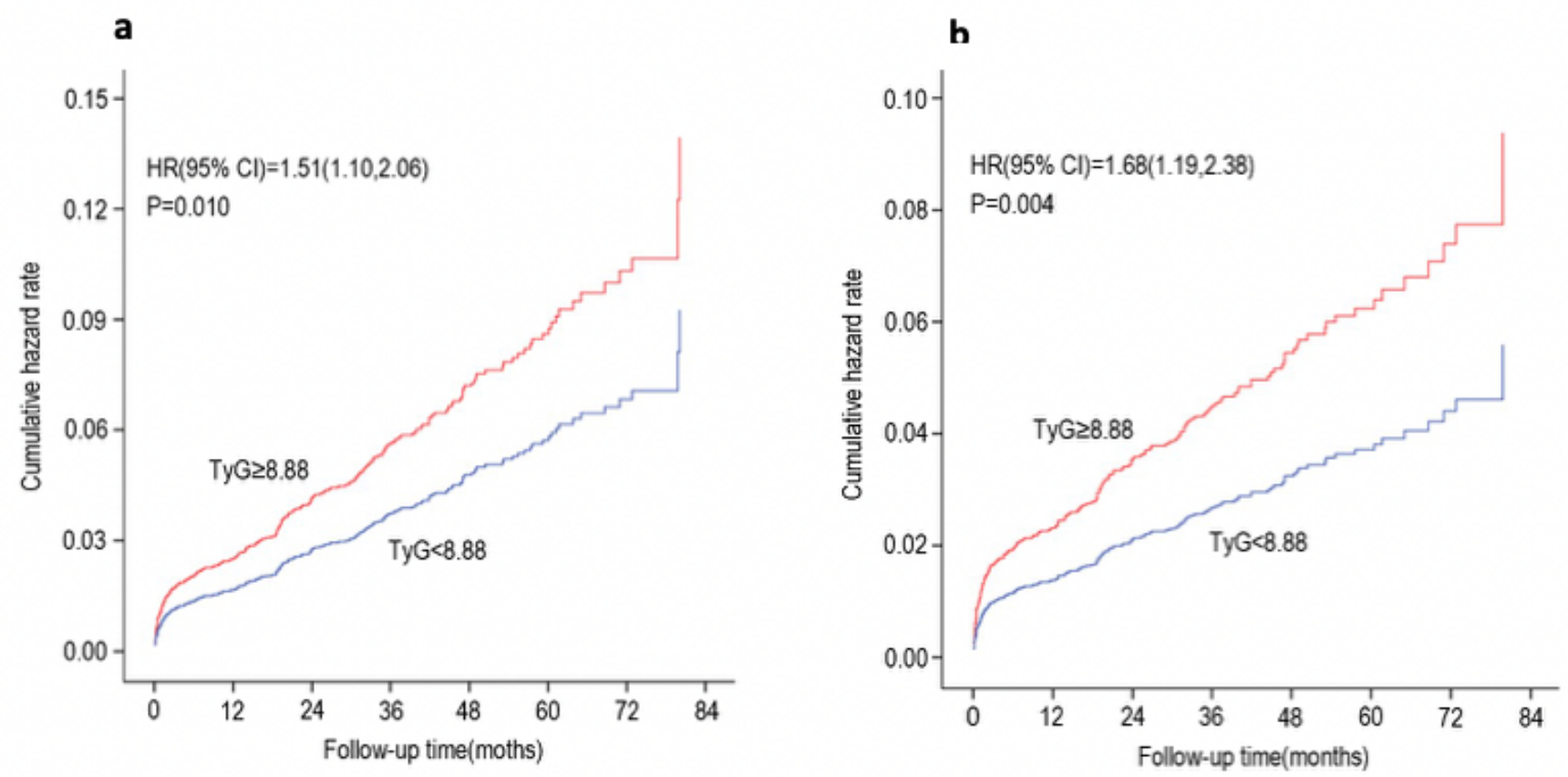

Figure 3

Cumulative hazard curves for all-cause death(a) and cardiac death(b) of the $T y \mathrm{G}<8.88$ group(blue line) versus the $T y G \geq 8.88$ group(red line). Adjusted for age, gender, diabetes mellitus, hypertension, previous AMI, hemoglobin, albumin, eGFR, TGs, LVEF and multi-vessel/LM. TyG, triglyceride-glucose index; AMI, acute myocardial infarction; eGFR, estimated glomerular filtration rate; TGs, triglycerides; LVEF: left ventricular ejection fraction; $\mathrm{LM}$, left main coronary artery; $\mathrm{HR}$, hazard ratio; $\mathrm{Cl}$, confidence interval. 

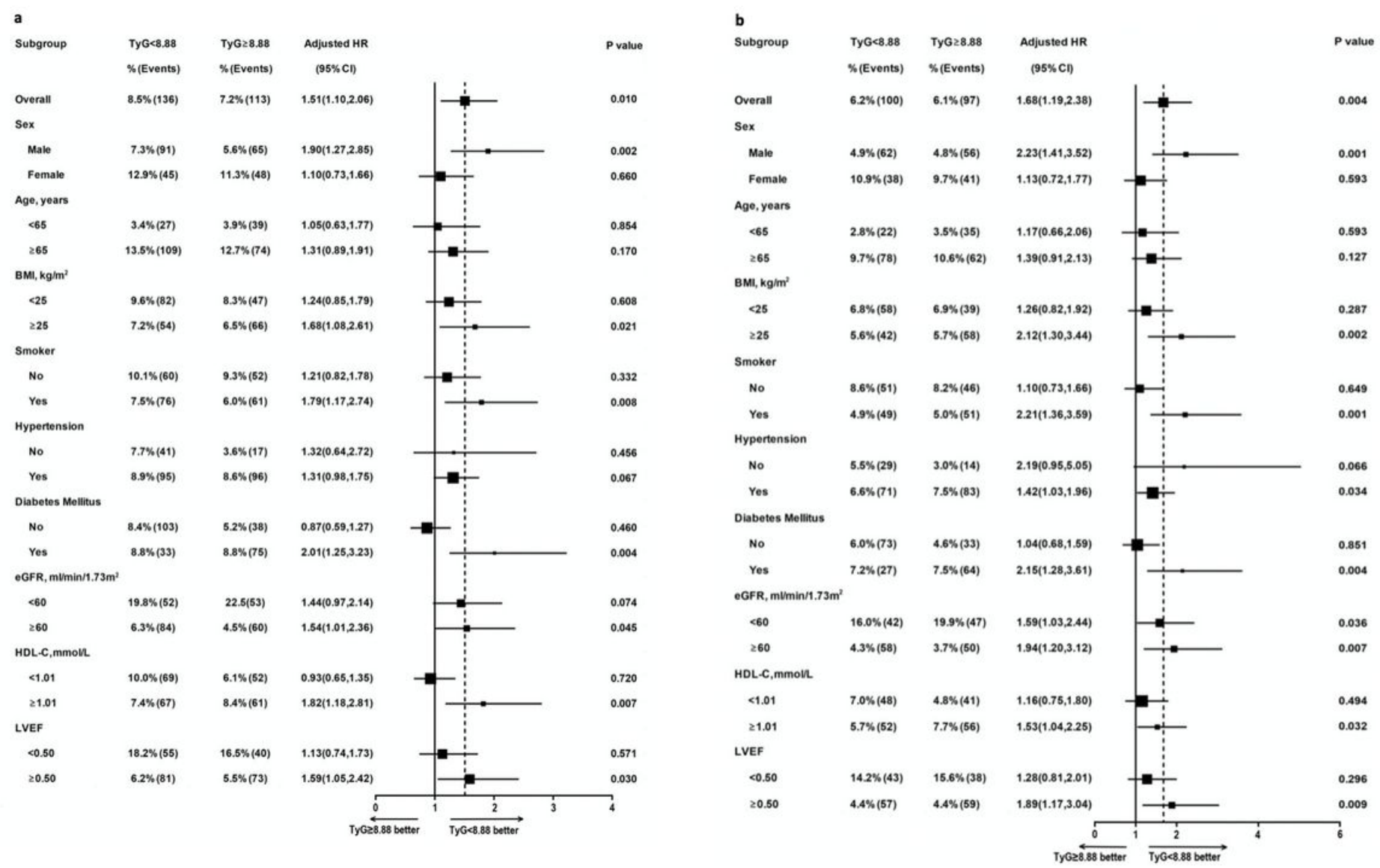

\section{Figure 4}

Forest plot of all-cause death(a) and cardiac death(b) according to different subgroups. Adjusted model included age, gender, diabetes mellitus, hypertension, previous AMI, hemoglobin, albumin, eGFR, TGs, LVEF and multi-vessel/LM. The dashed vertical line represents the hazard ratio for the overall study population. The box sizes are proportional to the precision of the estimates (with larger boxes indicating a greater degree of precision). TyG, triglyceride-glucose index; AMI, acute myocardial infarction; eGFR, estimated glomerular filtration rate; TGs, triglycerides; LVEF: left ventricular ejection fraction; LM, left main coronary artery; $\mathrm{HR}$, hazard ratio; $\mathrm{Cl}$, confidence interval. 